\title{
Mathematical theory of the duality computer in the density matrix formalism
}

\section{Gui Lu Long}

Published online: 1 July 2008

(C) Springer Science+Business Media, LLC 2008

Erratum to: Quantum Inf Process (2007) 6(1):49-54

DOI 10.1007/s11128-006-0042-1

The correct details of Ref. [2] in the above paper should be: G. L. Long, Commun. Theor. Phys. 45, 825 (2006), also available in arix:quant-ph/0512120.

The online version of the original article can be found under doi:10.1007/s11128-006-0042-1.

G. L. Long $(\bowtie)$

Key Laboratory of Atomic and Molecular Nanosciences, Department of Physics,

Tsinghua University, Beijing 100084, People's Republic of China

e-mail: gllong@mail.tsinghua.edu.cn 